Informal Report

\title{
On Plasma Transport and Flow \\ in the Fluid Picture
}

$\frac{\pi}{5}$

to

$\frac{7}{\omega}$

$\stackrel{\$}{\perp}$

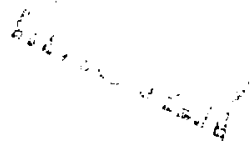


LA.7429.MS

Informal Report

UC-20f

Issued: August 1978

\section{On Plasma Transport and Flow in the Fluid Picture}

Guthrie Miller

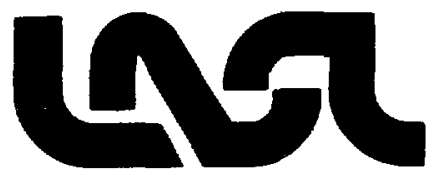


ON PLASMA TRANSPORT AND FLOW IN THE FLUID PICTURE

by

Guthrie Mil1er

ABSTRACT

The fluid equations are applied to cylindrical pinches, and the role of resistivity, heat conductivity, and viscosity is examined. Analytical solutions for spectal cases are reviewed. One-dimensional partial differential equations suitable for numerlcal solution are derived that describe: 1) the radial evolution of a pinch and 2) theta-pinch end loss (for $r=0$ ).

\section{INTRODUCTION}

The flutd model offers the slmplest means for a quantitative degcription of plasma behavior. In this report the flutd model is applied to cylindrical pinches (wostly to the theta pinch). The complete set of equations is too complicated for a general analytical solution, but in a few special cases this Is possible, and the analytical solutions known to the author are collected here. Analytical solutions are heurlstically useful and are also useful as check cases for numertcal solutions.

The main object in this report has been to derive tractable one-dimenstonal partial differential equations. Nowadays, with the use of computers, if a problem can be so formulated, it can be regarded as solved. The other object was to review the Implications of the fluld plcture, Including the role of transport coefficients. Viscosity, In part1cular, 1o rather 
complicated and this has led to a tendency to ignore it. In this report two cases where classical viscosity is of importance but that do not seem widely known are pointed out. These are: 1) for flow in the $z$ direction with long mean free path (end loss or $m=0$ oscillations) and 2 ) for long wavelength $m>2$ flow. For the second case it 1s shown that classical viscosity gives "finte-Larmor-radius" stabilization identical to that obtained from vlasov-fluid theory.

II. FLUID MODEL

Starting wth the fluid equations for a simple $Z=1$ plasma as given, for example, by Braginski1, 1 the quasi-neutral approximation $n_{e}=n_{1}=n$ is used, and the electron inertia and viscosity terms are neglected. Because $v_{e}=$ $v_{1}-f /(e n)$, where $f=\nabla \times B$, there are then nine variables: $n, v=v_{1}, s_{e}, s_{1}$ (entroples per particle for electrons and ions), and B (magnetic field). These are governed by the following nine equations.

$$
\left.\frac{\partial \mathbf{n}}{\partial t}\right|_{\mathbf{r}_{0}}=-\mathbf{n} \nabla \cdot \mathbf{v}
$$

$\left.\operatorname{Mn} \frac{\partial v}{\partial t}\right|_{r_{0}}=-\nabla_{p}-\operatorname{div} \Pi+j \times B$,

$$
\begin{aligned}
& \left.\pi T_{1} \frac{\partial s_{1}}{\partial t}\right|_{r_{0}}=\operatorname{div}\left(k_{1} \nabla T_{1}\right)+\frac{3}{2} n \frac{T_{e}-T_{1}}{\tau_{e q}}-\Pi_{\alpha \beta} \frac{\partial v_{\alpha}}{\partial r_{\beta}}, \\
& \left.n T_{e} \frac{\partial s_{e}}{\partial t}\right|_{r_{0}}=\mathrm{d} I v\left(\kappa_{e} \nabla T_{e}\right)+\frac{3}{2} n \frac{T_{1}-T_{e}}{\tau_{e q}} \\
& +\frac{T_{e}}{e} \jmath \cdot \nabla s_{e}+j \cdot \sigma^{-1} \jmath,
\end{aligned}
$$


$n \frac{\partial}{\partial t}\left(\frac{B}{n}\right)_{r_{0}}=B \cdot \nabla v-\nabla \times\left\{\sigma^{-1} j+\frac{f \times B-\nabla P_{e}}{e n}\right\}$.

Equations (7-9) are obtained from the equation of motion of the electron fluld by solving for the electric fleld $E$, taking the curl, and using $\nabla \times E=-\partial B / \partial t$.

The time derivatives are taken with the variable $r_{0}$ fixed, where $r_{0}$ is the initial coordinate of a fluid element. The relation between $r_{o}$ and $r$ is as follows:

$$
\begin{aligned}
& r=r_{0}+\int v d t \\
& \left.\frac{\partial}{\partial t}\right|_{r_{0}}=\left.\frac{\partial}{\partial t}\right|_{r}+v \cdot \nabla
\end{aligned}
$$

The reason for using $r_{0}$ as a variable 1 s that Eq. (1) 1s automatically satisfied for $n=n_{0}\left(d V_{0} / d V\right)$, where $n_{0}$ is the Initial density and $\left(d V_{0} / d V\right)$ the Jacobian of the transformation between $r_{0}$ and $r$. This can be seen mathematically as follows:

$$
\begin{aligned}
J \equiv \frac{d V}{d v_{0}} \equiv \frac{\partial(x, y, z)}{\partial\left(x_{0}, y_{0}, z_{0}\right)} & \\
\left.\frac{\partial J}{\partial t}\right|_{r_{0}} & =\frac{\partial\left(v_{x}, y, z\right)}{\partial\left(x_{0}, y_{0}, z_{0}\right)}+\frac{\partial\left(x, v_{y}, z\right)}{\partial\left(x_{0}, y_{0}, z_{0}\right)}+\frac{\partial\left(x, y, v_{z}\right)}{\partial\left(x_{0}, y_{0}, z_{0}\right)} \\
& =\left(\frac{\partial v_{x}}{\partial x}+\frac{\partial v_{y}}{\partial y}+\frac{\partial v_{z}}{\partial z}\right) \frac{\partial(x, y, z)}{\partial\left(x_{0}, y_{0}, z_{0}\right)}=\nabla \cdot v J
\end{aligned}
$$

Therefore,

$$
\left.\frac{\partial \mathbf{n}}{\partial t}\right|_{r_{0}}=\frac{\partial}{\partial t}\left(\frac{n_{0}}{J}\right)_{r_{0}}=-\left.\frac{n_{0}}{J^{2}} \frac{\partial J}{\partial t}\right|_{r_{0}}=-n \nabla \cdot v
$$


The electron and ton pressure and temperature are obtained from the entropy per particle using the perfect gas relation $T=[n \exp (s)]^{2 / 3}$. Braginsk11's notation for the varlous transport coefficients is followed with the exception that $\tau_{e q} \equiv M \tau_{e} /(2 m)$. Also note that $K_{1}, K_{e}, I$, and $\sigma^{-1}$ are tensor quantities depending on the magnetic fleld direction. For simplicity, the thermal force and additional terms in the electron and ion heat fluxes are ignored.

The strict conditions for validity of these equations are $t>\tau_{e}, \tau_{1}$, where $t$ Is a characteristic t1me, and $L>\lambda_{e}, \lambda_{1}$, where $L$ is a characteristic length. However, the fluid equations are used in practice (perhaps for good reason) when these condftions are violated.

\section{RADIAL EYOLUTION OF A PINCH}

In this section configurations having a dependence only on $r$ and $t$ are considered. These configurations are described by $n(r), v_{r}(r), s_{1}(r), s_{e}(r)$, $B_{z}(r)$, and $B_{\theta}(r)$. The case with $v_{\theta}(r) \neq 0$ is taken up briefly at the end of this section. The configuration is assumed basically to be in equilibrium (except for slow changes of the applied flelds and transport processes) and therefore the acceleration terms in Eqs. $(2-4)$ are dropped. The equations giving the time evolution of the system are as follows:

$$
\left.n T_{1} \frac{\partial s_{1}}{\partial t}\right|_{r_{0}}=\frac{1}{r} \frac{\partial}{\partial r}\left(r \kappa_{1} \frac{\partial T_{1}}{\partial r}\right)+\frac{3}{2} n \frac{T_{e}-T_{1}}{\tau_{e q}},
$$

$$
\begin{aligned}
\left.n T_{e} \frac{\partial s_{e}}{\partial t}\right|_{r_{0}} & =\frac{1}{r} \frac{\partial}{\partial r}\left(r \kappa_{e} \frac{\partial T_{e}}{\partial r}\right)+\frac{3}{2} n \frac{T_{1}-T_{e}}{T_{e q}} \\
& +\left[\frac{1}{r} \frac{\partial}{\partial r}\left(\mathrm{rB}_{\theta}\right)\right]^{2}\left(\frac{B_{z}^{2}}{B^{2} \sigma_{i}}+\frac{B_{\theta}^{2}}{B^{2} \sigma_{\perp}}\right)
\end{aligned}
$$




$$
\begin{aligned}
& +\left(\frac{\partial \mathrm{B}_{z}}{\partial r}\right)^{2}\left(\frac{\mathrm{B}_{z}^{2}}{\mathrm{~B}^{2} \sigma_{\perp}}+\frac{\mathrm{B}_{\theta}^{2}}{\mathrm{~B}^{2} \sigma_{A}}\right) \\
& -\frac{2}{r} \frac{\partial}{\partial r}\left(r \mathrm{~B}_{\theta}\right) \frac{\partial \mathrm{B}_{z}}{\partial \mathrm{r}} \frac{\mathrm{B}_{\theta} \mathrm{B}_{z}}{\mathrm{~B}^{2}}\left(\frac{1}{\sigma_{1}}-\frac{1}{\sigma_{1}}\right),
\end{aligned}
$$

$$
\begin{aligned}
\frac{\partial}{\partial t}\left(\frac{B_{z}}{n}\right)_{r_{0}} & =\frac{1}{n r} \frac{\partial}{\partial r}\left[r \frac{\partial B_{z}}{\partial r}\left(\frac{B_{z}^{2}}{B^{2} \sigma_{1}}+\frac{{ }^{B}{ }^{2}}{B^{2} \sigma_{1}}\right)\right. \\
& \left.-\frac{\partial}{\partial r}\left(r_{\theta}\right) \frac{B_{\theta} B_{z}}{B^{2}}\left(\frac{1}{\sigma_{1}}-\frac{1}{\sigma_{1}}\right)\right],
\end{aligned}
$$

$$
\begin{aligned}
\frac{\partial}{\partial t}\left(\frac{{ }^{B} \theta}{n r}\right)_{r_{0}} & =\frac{1}{n r} \frac{\partial}{\partial r}\left[\frac{1}{r} \frac{\partial}{\partial r}\left(r_{\theta}\right)\left(\frac{B_{z}^{2}}{B^{2} \sigma_{1}}+\frac{B_{\theta}^{2}}{B^{2} \sigma_{\perp}}\right)\right. \\
& \left.-\frac{\partial B_{z}}{\partial r} \frac{{ }^{B} \theta_{z} B_{z}}{B^{2}}\left(\frac{1}{\sigma_{1}}-\frac{1}{\sigma_{1}}\right)\right]
\end{aligned}
$$

The equilibrium relation from Eqs. (2-4) is

$$
\frac{\partial}{\partial r}\left(p+\frac{B^{2}}{2}\right)+\frac{B_{\theta}^{2}}{r}=0
$$

At any instant of time this equation and the continufty relation, $\partial r^{2} / \partial r_{0}{ }^{2}=$ $n_{0} / n$, are two equations that allow $r\left(r_{0}\right)$ and $n(r)$ to be determined from the known values of $s_{1}, s_{e}, B_{z} / n$, and $B_{\theta} /(n r)$.

For the low-pressure case, $p \ll B^{2}$, with $\sigma_{1}=\sigma_{1}=$ const, there is an Interesting analytic solution of Eqs. (13) and (14) given by 


$$
\begin{aligned}
& B_{z}=B_{0} J_{0}(k r) \exp \left(-k^{2} t / \sigma\right), \\
& B_{\theta}=B_{0} J_{1}(k r) \exp \left(-k^{2} t / \sigma\right),
\end{aligned}
$$

wh $n(r)$ constant in time.

Only the case $B_{\theta}=0$ (theta pinch) w11l be considered further. In this case the equilibrium relation is

$$
p+\frac{B^{2}}{2}=\frac{B e^{2}}{2},
$$

where $B_{e}$ is the external magnetlc field far from the plasma. Equation (16) by 1tself determines $n\left(r_{0}\right)$, with $r\left(r_{0}\right)$ then obtalned using $\partial r^{2} / \partial r_{0}{ }^{2}=n_{0} / n$.

The simplest special care of Eqs.(11-14) is when all terms on the right-hand sides are small (e.g., for high temperatures). In this case $s_{i}, s_{e}$, and $B_{z} / n$ are constants on-axis, since $v_{r}=0$ for $r=0$. The conditions on-axis during adlabatic compression or expansion are determined from Eq ( 16 ),

$$
B_{0}\left(\frac{n}{n_{0}}\right)^{5 / 3}+\left(1-B_{0}\right)\left(\frac{n}{n_{0}}\right)^{2}=\left(\frac{B}{B_{0}}\right)^{2}
$$

where $B_{0}$ is the initial beta on-axis, $n_{0}$ the inftial density on-axis, and $B_{0}$ the Inftial applied magnetic field.

It is useful to make order-of-magnitude estimates for the various transport processess described on the right-hand sides of Eqs.(11-14). The electrons and lons cool by radial heat conduction in times $t$ $\simeq \mathrm{na}^{2} / \mathrm{k} \sim \mathrm{a}^{2} \mathrm{a}^{2} \mathrm{~T}^{1 / 2} /\left(\mathrm{nm}^{1 / 2}\right){ }^{*}$ These times become infinite if the plasma is surrounded by a vacuum region $(n * 0)$. Also, Ion heat conduction is a factor $(M / m)^{1 / 2}$ more 1mportant then electron heat conduction. The electron and ion temperatures approach a common value in a time $t=\tau_{e q} \sim T_{e}{ }^{3 / 2}$. Finfte

*wT $\gg 1$ is assumed throughout this report. 
cunductivity apparently causes field diffusion with a characteristic time $t$ $\simeq a^{2} \sigma_{\perp} \sim a^{2} T_{e}^{3 / 2}$.

To more carefully examine diffusion, a useful first approximation is to assume that the conductivity is constant. This corresponds to a flat initial $\mathrm{T}_{\mathrm{e}}$ profile and short times so that resistive heating is small. The latter requirement is not restrictive since the time for resistive heating is the same as the time for the plasma to diffuse away. The time $t$ for apprectable heating is determined by $j^{2} t / \sigma \simeq p$, where $j B \simeq p / a$ so that $t \simeq \sigma a^{2}{ }^{2} / p$, whfch is the diffusion time ( $B$ is the external field), as will be seen later.

For constant temperature the diffusion process is described by the simple equation,

$$
\frac{\partial}{\partial t}\left(\frac{{ }^{B} z}{n}\right)_{r_{o}}=\frac{1}{\sigma n r} \frac{\partial}{\partial r}\left(r \frac{\partial B_{z}}{\partial r}\right)
$$

A further fairly good approximation, due to the fact that $v_{r}=0$ at $r=0$, is to take $\partial / \partial t\left|r_{0} \simeq \partial / \partial t\right| r$. There are two simple limiting cases: 1 ) high $B$, where $n \simeq$ const, which gives

$$
\frac{\partial B_{z}}{\partial t}=\frac{1}{\sigma r} \frac{\partial}{\partial r}\left(r \frac{\partial B_{z}}{\partial r}\right)
$$

and a characteristic diffusion time $\sigma a^{2}$, where a is the plasma characteristic radius, and 2) low $B$, where $B=$ const, which gives (using $\partial B / \partial r=-1 / B \partial p / \partial r$ )

$$
\frac{\partial n}{\partial t}=\frac{p}{B^{2} \sigma r} \frac{\partial}{\partial r}\left(r \frac{\partial n}{\partial r}\right),
$$

and a characteristic diffusion time $\sigma a^{2}{ }^{2} / p$. This time with $B$ the external field applies for both high and low beta since in the high-beta case $B^{2} / p \simeq 1$. Order-of-magnttude estimates can be given for the importance on the diffusion time scale of the terms neglected In Eqs. (7-9). These terms are 1) electron inertia $\left.\simeq\left(m / e^{2} n\right) / a^{2}\left(p / B^{2}\right), 2\right)$ electron $v 1 s \cos 1 t y=\left(m / e^{2} n\right) / a^{2}\left(n T_{e} / B^{2}\right)$, 
and 3) thermal force $=n T_{e} / p$. Although not clear from this rough estimate, the thermal force is not of 1mportance in theta plnches with $T_{e}<T_{1}$ and moderate temperature gradlents.

Finally, if $v_{\theta} \neq 0$ the $r$ component of Eqs.(2-4) becomes

$$
\frac{\partial}{\partial r}\left(p+\frac{B^{2}}{2}\right)-\frac{M n v_{\theta}^{2}}{r}=0
$$

The centrifugal force term is only aignificant if $\mathrm{Nuv}_{\theta}{ }^{2} \approx \mathrm{p}$ or $\mathrm{v}_{\theta} \approx$ thermal speed, which means that the Ion Larmor orbits are significantly displaced during one perlod. Rotation-driven Instabilities become important for smaller rotation speeds ${ }^{2}$ so this term is usually dropped.

The $\theta$-component of Eqs. (2-4), which describes the viscous damping of the Ion rotation, 18

$$
-M n \frac{\partial v_{\theta}}{\partial t}=\frac{1 \partial}{r^{2 \partial r}}\left(r^{2} \Pi_{r \theta}\right)
$$

From Braginsk11, 1 the $r, \theta$ component of the viscous stress tensor 18 given by

$$
\pi_{\mathbf{r} \theta}=-\eta_{1}\left(\frac{\partial v_{\theta}}{\partial \mathbf{r}}-\frac{\mathbf{v}_{\theta}}{\mathbf{r}}\right)+\eta_{3}\left(\frac{\partial v_{\mathbf{r}}}{\partial \mathbf{r}}-\frac{\mathbf{v}_{\mathbf{r}}}{\mathbf{r}}\right)
$$

The effect of the $n_{3}$ viscosity component (gyro-viscosity) will be discussed later on, and if this term is simply 1gnored there results an equation for $v_{\theta}$,

$$
\sin \frac{\partial}{\partial t}\left(\frac{v_{\theta}}{r}\right)=\frac{1}{r^{3}} \frac{\partial}{\partial r}\left[n_{1} r^{3} \frac{\partial}{\partial r}\left(\frac{v_{\theta}}{r}\right)\right]
$$

An order-of-magnitude estimate for the time for the lon velocity distribution to decay to a $\mathbf{r} 1 \mathrm{gid}$ rotation is 


$$
\mathrm{t}=\frac{10}{3} \frac{\mathrm{Mna}^{2}}{\eta_{1}}=\frac{\omega_{1}{ }^{2} \mathrm{a}^{2} \mathrm{M} \tau_{1}}{\mathrm{~T}_{1}} \sim \mathrm{B}^{2} \mathrm{a}^{2} \mathrm{~T}^{1 / 2} /\left(\mathrm{nM}^{1 / 2}\right)
$$

IV. LONGITUDINAL FLOW AND TRANSPORT

The main confinement limitation for the $\theta$ pinch is flow out the ends due to lack of equilibrium. Longitudinal heat conduction may also be of importance (e.g., with end plugs). The situation 1 s actually two-dimensional $(z$ and $r$ ) but useful one-dimensional equations can be obtained by neglecting radial transport and considering only the axis of the system $(r=0)$. These equations are as follows:

$\left.\operatorname{Mn} \frac{\partial^{2} z}{\partial t^{2}}\right|_{z_{0}}=-\frac{\partial p}{\partial z}+\frac{4}{3} \frac{\partial}{\partial z}\left(\eta_{0} \frac{\partial v_{z}}{\partial z}\right)$,

$$
\left.n T_{1} \frac{\partial s_{1}}{\partial t}\right|_{z_{0}}=\frac{\partial}{\partial z}\left(\kappa_{1} \frac{\partial T_{1}}{\partial z}\right)+\frac{3}{2} n \frac{T_{e}-T_{1}}{\tau_{e q}}+\frac{4}{3} n_{0}\left(\frac{\partial v_{z}}{\partial z}\right)^{2},
$$

$$
\left.n T_{e} \frac{\partial s}{\partial t}\right|_{z_{o}}=\frac{\partial}{\partial z}\left(k_{e} \frac{\partial T_{e}}{\partial z}\right)+\frac{3}{2} n \frac{T_{1}-T_{e}}{\tau_{e q}}
$$

From the continuity equation

$$
n=\frac{n_{0}}{\frac{\partial\left(z, x^{2}\right)}{\partial\left(z_{0}, z_{0}^{2}\right)}}
$$

9 
with

$$
\frac{\partial\left(z, r^{2}\right)}{\partial\left(z_{0}, r_{0}^{2}\right)}=\left(\frac{\partial z}{\partial z_{0}}\right)\left(\frac{\partial r^{2}}{\partial r_{0}^{2}}\right)
$$

for $r=0$. The radial equilibrium relation 18 needed to determine $\partial \mathrm{r}^{2} / \partial \mathrm{r}_{0}{ }^{2}$ and close the system,

$$
\frac{n_{0} 5 / 3\left[\exp \left(\frac{2}{3} \theta_{e}\right)+\exp \left(\frac{2}{3} \beta_{1}\right)\right]}{\left(\frac{\partial z}{\partial z_{0}}\right)^{5 / 3}\left(\frac{\partial r^{2}}{\partial r_{o}{ }^{2}}\right)^{5 / 3}}+\frac{B_{o}^{2}\left(1-B_{o}\right)}{2\left(\frac{\partial r^{2}}{\partial r_{o}{ }^{2}}\right)^{2}}=\frac{B_{e}{ }^{2}}{2},
$$

where $B_{0}$ is the 1nitial value of the external field $B_{e}$, and $B_{0}$ the inftal beta value on-axis.

Neglecting heat conduction, equilibration of electrons and 1ons, and ton viscosity, and assuming small ampt1tude disturbances $1 \cdot e \cdot, \quad \partial z / \partial z_{0}=1+\varepsilon$, and constant external field, Eq. (17) becomes

$$
\frac{\partial^{2} \varepsilon}{\partial t^{2}}=-\frac{1}{M_{0}} \frac{\partial^{2} p}{\partial z^{2}}=-\frac{c^{2} u_{A}^{2}}{c^{2}+u_{A}^{2}} \frac{\partial^{2} \varepsilon}{\partial z^{2}} .
$$

The small signal propogation velocity on-axis is thus $v^{2}=c^{2} u_{A}^{2} /\left(c^{2}+u_{A}{ }^{2}\right)$, where $c^{2}=5 \mathrm{p}_{\mathrm{o}} /\left(3 \mathrm{Mn}_{\mathrm{o}}\right)$ and $\mathrm{u}_{\mathrm{A}}{ }^{2}=\left(1-\mathrm{B}_{\mathrm{O}}\right) \mathrm{B}_{\mathrm{O}}{ }^{2} /\left(\mathrm{Mn}_{\mathrm{O}}\right)$. Another analytic result can be obtained for a simple isentropic rarefaction in the low- $\beta$ 1imit. In this case $\partial r^{2} / \partial r_{0}{ }^{2}=1$ and $n=n_{0} \partial z_{o} / \partial z$. The equation of motion, Eq. (17), is then

$$
\left.\frac{\partial^{2} z}{\partial t^{2}}\right|_{z_{0}}=-\frac{1}{n_{0}}-\frac{\partial p}{\partial z_{o}}
$$


Taking derivatives with respect to $z_{0}$, this becomes

$$
\frac{\partial^{2}}{\partial t^{2}}\left(\frac{n_{0}}{n}\right)_{z_{0}}=\frac{\partial^{2}}{\partial t^{2}}\left(\frac{p_{o}}{p}\right)_{z_{0}}^{3 / 5}=-\frac{1}{M_{0}} \frac{\partial^{2} p}{\partial z_{o}^{2}}
$$

The solution* of this equation for $p t_{t=0}=F(z)$ is

$$
p=F\left(z_{0} \pm c_{0}\left(\frac{p}{p_{0}}\right)^{4 / 5} t\right)
$$

where $c_{0}^{2}=5 p_{0} /\left(3 M_{0}\right)$. For a situation with the pressure inftially constant from $z=0$ to $z=L$ the pressure takes the value $p$ at point $z_{0}$ given by

$$
z_{0}=L-c_{0}\left(\frac{p}{P_{0}}\right)^{4 / 5} t
$$

therefore,

$$
p=p_{0}\left(\frac{L-z_{0}}{c_{0} t}\right)^{5 / 4}
$$

The complete solution is obtained using

$$
z=L-c_{0} t+\int_{L-c_{0} t}^{z_{0}}\left(\frac{n_{0}}{n}\right) d z_{0}=z_{1}+\int_{z_{1}}^{z_{0}}\left(\frac{P_{0}}{p}\right)^{3 / 5} d z_{0},
$$

$\star_{A}$ solution of the equation $\partial^{2} f / \partial x^{2}=\alpha \partial^{2} f^{\lambda} / \partial y^{2}$ is $E=F\left(y \pm(\alpha \lambda)^{1 / 2} f(\lambda-1) / 2 x\right)$, where $F$ is an arbitrary function. As can be seen $F(y)$ gives the value of $f(x, y)$ for $x=0$. 
where $z_{l} \equiv L-c_{0} t$, which \&1ves

$$
\left(\frac{l-z_{0}}{c_{0} t}\right)^{1 / 4}-1-\left(\frac{z-z 1}{4 c_{0} t}\right)
$$

and thus

$$
p=\left\{\begin{array}{l}
p_{0}, \quad 0<z<z_{1}, \\
p_{0}\left[1-\frac{z-z_{1}}{4 c_{0} t}\right]^{5}, \quad z>z_{1}
\end{array} .\right.
$$

The propagation velocity of the rarefaction $\left(c_{0}\right)$ agrees with the small signal velocity $\left(v=c\right.$ for $\left.u_{A} \rightarrow \infty\right)$.

It is important to note that a quantitative treatment of the flow requires a two-dimensional treatment. Figure 1 gives the radially integrated plasma density historles at varlous points in a theta pinch of half-length $L$ as calculated by Brackbill for ideal two-dimenstonal flow. The rarefaction propagation velocity is $\mathrm{v}=1.7 \mathrm{v}_{i}$ from $\mathrm{Fig} \cdot 1 \quad\left(\mathrm{~T}_{\mathrm{e}}=\mathrm{T}_{1}, \quad B=0.7, \quad \mathrm{v}_{1} \equiv\left(2 \mathrm{~T}_{1} / \mathrm{M}^{1 / 2}\right)\right.$ which is quite different from that given by Eq. (22), $v=0.75 v_{1}$.

Order-of-magnitude estimates of the transport terms In Eqs. (17-19) can be made in a straightforward way. One observation is that the times assoclated with viscous damping and with ion heat conduction are of the same order and are given by $t=L 2 /\left(v_{1} \lambda_{1}\right)$, where $\lambda_{1}$ is the Ion mean free path and $v_{1}$ the ton thermal velocity. Thus these processes are of dominating importance in simple end $108 s\left(t<L / v_{1}\right)$ only for $\lambda_{1}>L$, which violates the original assumption on which the flutd description is based. Also, with $\lambda_{1}>L$, the heat and monentum fluxes exceed their kinetic limits $n \mathrm{nv}_{1}$ and $\mathrm{nMv}_{1}{ }^{2}$. The fluld equations are commonly used to describe situations with long mean free path, and in these cases the fon heat flux and momentum flux (perhaps with flux limits applied) are of Importance. 
There are several analytical solutions for longltudinal heat conduction. The first (DeSilva, Chu, and Johnson) covers the interesting situation $\partial \mathrm{p} / \partial z=0$ and $T_{e}=T_{1}$. Neglecting ton heat conductivity, Eqs.(18) and (19) become in the case

$$
2 n T \frac{\partial g}{\partial t}=\frac{\partial}{\partial z}\left(\kappa_{e} \frac{\partial T}{\partial z}\right)
$$

where $s=\log \left(\mathrm{T}^{3 / 2} / \mathrm{n}\right)$, and $\mathrm{k}_{\mathrm{e}}=\alpha \mathrm{T}^{5 / 2}$ with $\alpha=$ const. A solution is

$$
T=T_{0}\left[1-(z / L)^{2}\right]^{2 / 7} f(t)
$$

with

$$
f(t)=\left[1+1.24 \frac{10 \alpha T_{0}^{5 / 2}}{21 n_{0} L^{2}} t\right]^{-2 / 5 *}
$$

The density profile is constant in time,

$$
n(z)=\frac{n_{0}}{1.24}\left[1-(z / L)^{2}\right]^{-2 / 7},
$$

and satisfies

$$
\int_{0}^{L} n d z=n_{0} L
$$

*If the electron-ion equilibration time 18 long, these formulas with $t+2 t$ describe the decay of the electron temperature. 
Another solution (Drelcer, Chu, and Johnson) covers the care $n=$ const and is interesting as an 1llustration of the small effect of the density profile. For this case Eq. (24) becomes

$$
3 n \frac{\partial T}{\partial t}=\alpha \frac{\partial}{\partial z}\left(T^{5 / 2} \frac{\partial T}{\partial z}\right)
$$

which is satisfied by

$$
T=T_{0} f(z)^{2 / 7}\left(1+2.13 \frac{5 \alpha T_{0}^{5 / 2}}{21 n L^{2}} t\right)^{-2 / 5}
$$

where

$$
\frac{d^{2} f}{d z^{2}}=-\frac{2.13}{L^{2}} f^{2 / 7}
$$

and $f(0)=1, f(L)=0$. Since $f^{2 / 7} \simeq 1, f \simeq 1-(z / L)^{2}$.

There is a non-separable solution of the electron heat conduction problem which describes the establishment of a temperature profile when a column with inftial temperature $T_{0}$ is connected to a cold heat sink at one end. This is given by

$$
T=T_{0} f\left[\left(\frac{z^{2} n}{\alpha T_{0}^{5 / 2} t}\right)^{1 / 2}\right]
$$

where $f(x)$ is determined from the equation 


$$
\frac{2}{7} \frac{d^{2} f^{7 / 2}}{d x^{2}}+\frac{3}{4} x \frac{d f}{d x}=0 *
$$

with the boundary conditions $f(0)=0, f(\infty)=1$. The function $f$ is plotted in F1g. 2. The temperature profile for $x$ in the range 0 to 2 closely matches the shape $\left[1-(L-z)^{2} / L^{2}\right]^{2 / 7}$ for $z=x L / 2$; therefore, the time to set up a steady profile in a column of half-length $L$ is approximately $t=L^{2} n /\left(4 \alpha T_{o} 5 / 2\right)$. Chu and Johnson ${ }^{3}$ obtain a similar estimate with the 4 in the denominator replaced by 3.7. When thermal losses are important (e.g., $T_{e}$ large) this time tends to be shorter than the time for mechanical motion or electron-ton equilibration to take place, thus the assumptions of constant density and no equilibration are val1d.

It is possible to reduce Eqs.(18-19) to zero dimensions by assuming the temperature profiles are given by Eq. (25). By symmetry, $v_{z}=0$ at $z=0$, $\partial / \partial t\left|z_{0}=\partial / \partial t\right|_{z}$ and therefore, Eqs.(18-19) become ordinary differential equations describing the point $r=0, z=0$.

\section{GYRO-VISCOSITY}

In this section the effect of gyro-viscosity $\left(\eta_{3} \neq 0\right)$ on long wavelength small oscillations of a theta pinch is examined. This type of viscosity does not dissipate energy, yet it results in "finfte-Larmor-radius" stabilization of m>2 modes in a theta pinch as will be seen. Since the energy of motion does not die away with this type of viscosity, the stabilized modes must be purely oscillatory.

First, it will be shown that gyro-viscosity drops out of the equation of motion for small oscillations of a constant-pressure plasma (sharp-boundary model). Therefore, the viscosity enters in only through the boundary conditions at the plasma surface. For long wavelength motion the z-dependence Is small and the $x$ and $y$ components of the equations of motion, Eqs. (2-4), contaln additional terms owing to viscosity, e.8.,

$$
(\operatorname{div} \Pi)_{x}=\frac{\partial \Pi x x}{\partial x}+\frac{\partial \Pi x y}{\partial y}
$$

*The electron-fgn equilibration time is liere assumed to be long. If 1 it is short, $f(x)+f\left(2^{1 / 2} x\right)$. 


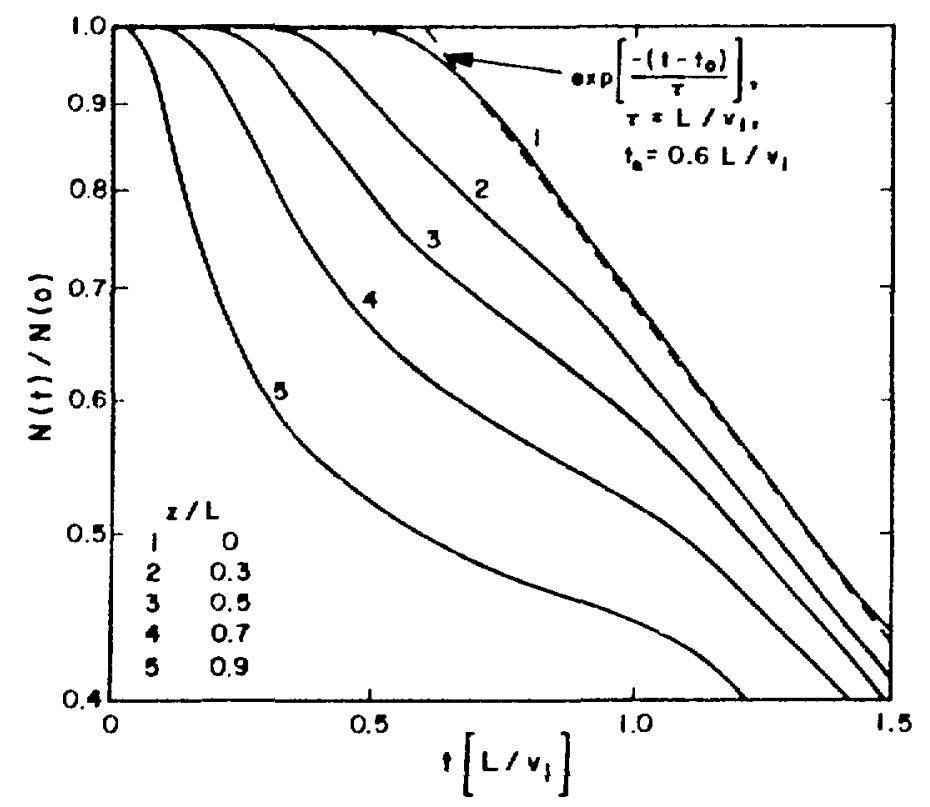

$\mathrm{F} 1 \mathrm{~g} \cdot 1$.

Line density histories at varlous points in a theta pinch of half-length $L$, from a two-dimensional computation by Brackbill assuming adlabatic flow.

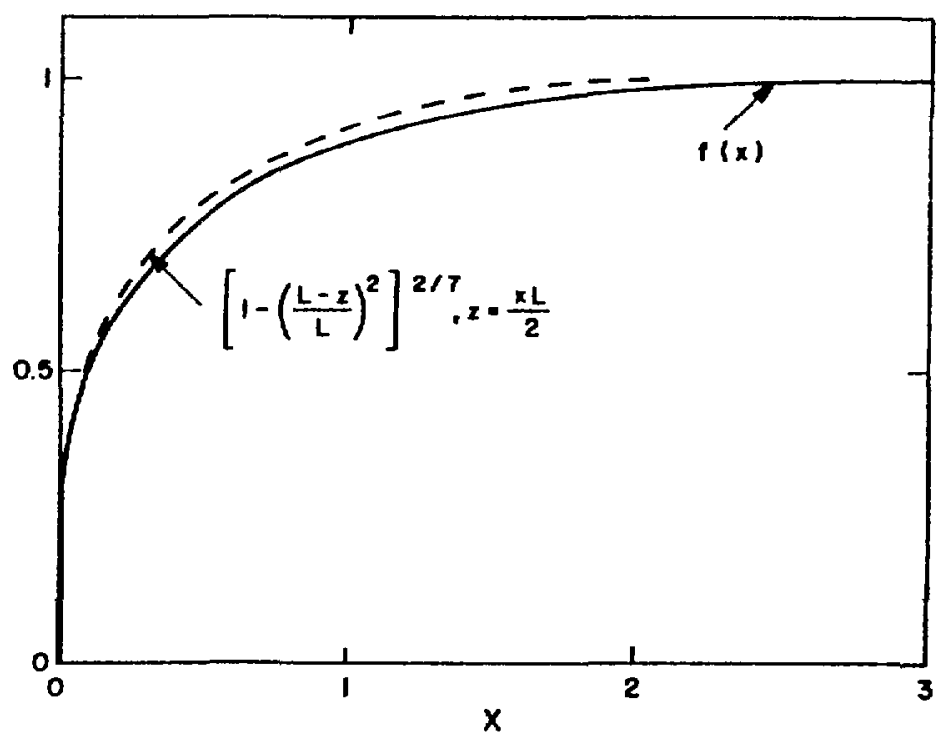

Fig. 2 .

A plot of the function $f(x)$, where $x=\left(z^{2} /(D t)\right)^{1 / 2}$, with $D$ the thermal diffusion coefficient, which describes the propagation of a "cooling wave". 
Because long wavelength motion is approximately divergenceless potential flow In the sharp-boundary model, 1.e., $v=\nabla \phi, \nabla^{2} \phi=0$, the additional viscosity terms are zero,

$$
(\operatorname{div} \pi)_{x}=n_{3}\left(\frac{\partial^{2} v_{y}}{\partial x^{2}}+\frac{\partial^{2} v y}{\partial y^{2}}\right)=0
$$

The boundary condition with viscosity is

$$
\left[p+\frac{B^{2}}{2}+\pi r r\right]_{\text {in }}=\left[\frac{B^{2}}{2}\right]_{\text {out }} \text {, }
$$

where

$$
\Pi_{r r}=-\eta_{3}\left(\frac{1}{r} \frac{\partial v_{r}}{\partial \theta}-\frac{v_{\theta}}{r}+\frac{\partial v_{\theta}}{\partial r}\right)
$$

To apply the boundary condition it is convenient to use a complex formalism, where

$$
\begin{aligned}
& \xi_{r}=\operatorname{Re}\left\{\left(\frac{r}{a}\right)^{m-1} \xi_{0} \exp [-1(m \theta+w t)]\right\} \\
& \xi_{\theta}=\operatorname{Re}\left\{-1\left(\frac{r}{a}\right)^{m-1} \xi_{0} \exp [-1(m \theta+\omega t)]\right\}
\end{aligned}
$$

with $\xi_{0}$ a complex number. This can be seen to represent divergenceless potentlal flow. The components of the boundary condition, Eq. (27), are as follows. $\left(\operatorname{Re}\left\{\xi_{0} \exp [-1(m \theta+\omega t)]\right\}\right.$ is factored out in each case): 


$$
\begin{aligned}
& \left(p+\frac{B^{2}}{2}\right)_{1 n}=\frac{\text { anM } \omega^{2}}{m} \\
& \left(\frac{B^{2}}{2}\right)_{\text {out }}=0,
\end{aligned}
$$

while using Eqs.(28) and (29) and $n_{3}=n T_{1} /\left(2 w_{1}\right)$,

$$
\pi_{r r}=\frac{(m-1) n T_{1}}{\omega_{1}^{a}} w
$$

where $\omega_{1}=e_{0}(1-\beta)^{1 / 2} / M$.

The boundary condition, Eq. (27), leads to the dispersion relation

$$
w^{2}+\frac{m(m-1) T_{1}}{\omega_{1} a^{2} M} w=0
$$

which is Identical to that obtained by Turner ${ }^{5}$. The viscosity term is roughly equal to $\omega \Omega *$, where $\Omega *$ is the dlamagnetic rotation frequency.

To appreciate the importance of viscosity one must imagine a situation that is otherwise unstable (because of additional magnetic flelds or rotation). The dispersion relation is then of the form

$$
\omega^{2}+\eta \omega+\gamma^{2}=0
$$

which has stable (purely oscillatory) roots for $\eta>2 \gamma$.

\section{REF ERENCES}

1. S. I. Braginski1, "Transport Processes In a Plasma", in Reviews of Plasma Physics, M. A. Leontovich, Ed. (Consultants Bureau, New York, 1965), Vol. I, P. 205. 
2. J. P. Freidberg and L. D. Pearlstein, "Rotational Instabilities in a Theta Pinch", Phys. Fluids 20, 1207 (1978).

3. T. K. Chu and L. C. Johnson, "Conduction Heat Loss Scaling in Open Field Line Geometries", Phys. Fluids 20, 1684 (1977).

4. G. Miller, "Motion of a Plasma Column in a Perturbing Magnetic Field", Phys. Flutds 18,1704 (1975).

5. L. Turner, "Finite-Larmor-Radius Stabilization in a Sharp-Boundary Vlasov-Fluid Screw Plnch", Phys. Flulds 20, 654 (1977). 